\title{
On partition functions of refined Chern-Simons theories on $S^{3}$
}

\author{
M.Y. Avetisyan and R.L. Mkrtchyan \\ Yerevan Physics Institute, \\ 2 Alikhanian Br. Str., 0036 Yerevan, Armenia \\ E-mail: maneh.avetisyan@gmail.com, mrl55@list.ru
}

ABSTRACT: We present a new expression for the partition function of the refined ChernSimons theory on $S^{3}$ with an arbitrary gauge group, which is explicitly equal to 1 when the coupling constant is zero. Using this form of the partition function we show that the previously known Krefl-Schwarz representation of the partition function of the refined Chern-Simons theory on $S^{3}$ can be generalized to all simply laced algebras.

For all non-simply laced gauge algebras, we derive similar representations of that partition function, which makes it possible to transform it into a product of multiple sine functions aiming at the further establishment of duality with the refined topological strings.

Keywords: Chern-Simons Theories, Topological Field Theories, Topological Strings

ARXiv EPrint: 2107.08679 


\section{Contents}

1 Introduction 1

2 The partition function of the refined CS theory on $S^{3} \quad 3$

3 Integral representation of the partition function for the refined CS theories

4 The partition function of the refined CS for simply laced algebras

5 On validity of (4.4) for all simply laced algebras

6 A universal-type presentation of the partition function for the non-simply laced algebras

\section{Introduction}

The partition function of Chern-Simons (CS) theory on a three-dimensional sphere $S^{3}$, first calculated in [1] (see below (2.1)), is presented in a universal form in [2,3], which means that alternative to the pure Lie algebra data - roots, invariant scalar product, etc., it is now expressed in terms of the so-called Vogel's universal parameters $\alpha, \beta, \gamma[4,5]$. These universal parameters are the homogeneous coordinates of a projective plane, which is called Vogel's plane in this context, so that special points in this plane correspond to all the simple Lie algebras (see Vogel's table 1). The projective nature of the universal parameters, i.e. their rescaling property, is the reflection of the possibility of rescaling of the invariant scalar product in the Lie algebra $[5,6]$.

The advantage of this representation is that it is very convenient for the further transformation of the abovementioned partition function into the Gopakumar-Vafa partition function of topological strings, as shown in $[3,7]$ for CS theory with the classical groups. In the recent work [8] this transformation has been extended to the CS with the exceptional groups, meaning that the partition function of CS on $S^{3}$ with an exceptional gauge group has been presented in the form of a partition function of a specific refined topological string. This should be considered as a step towards the establishment of the duality of the corresponding theories. The fact that all exceptional algebras (actually all algebras in $E_{8}$ row of the Freudenthal magic square) belong to a line in Vogel's plane - the so-called Deligne's line, is exploited in that work. Deligne [9] suggested that all the points on that line make up a so-called series of Lie algebras, which was partially confirmed in [10]. 


\begin{tabular}{|r|r|r|r|r|r|}
\hline & $\alpha$ & $\beta$ & $\gamma$ & $t=\alpha+\beta+\gamma$ & Line \\
\hline$s u(N)$ & -2 & 2 & $N$ & $N$ & $\alpha+\beta=0$ \\
\hline$s o(N)$ & -2 & 4 & $N-4$ & $N-2$ & $2 \alpha+\beta=0$ \\
\hline$s p(N)$ & -2 & 1 & $N / 2+2$ & $N / 2+1$ & $\alpha+2 \beta=0$ \\
\hline $\operatorname{Exc}(a)$ & -2 & $a+4$ & $2 a+4$ & $3 a+6$ & $\gamma=2(\alpha+\beta)$ \\
\hline
\end{tabular}

For the exceptional line $\operatorname{Exc}(a) a=-2 / 3,0,1,2,4,8$ for $G_{2}, \mathrm{SO}(8), F_{4}, E_{6}, E_{7}, E_{8}$, respectively.

Table 1. Vogel's parameters.

The main features of the presentation of the partition function discovered in $[2,3]$ has been extended to include the partition function of the refined CS theory on a $3 d$ sphere $^{1}$ for $A_{n}$ and $D_{n}$ algebras in [11]. It has also been shown to be very convenient for the derivation of the partition function of the dual refined topological strings in [12]. In the same work the non-perturbative corrections to the partition function of topological strings, derived from the universal CS partition function [3] (with $A_{n}$ gauge algebra), has been shown to coincide with those, derived in $[13,14]$ directly in the topological string theory framework, thus extending the CS/topological strings duality to the non-perturbative domain.

The natural development of these investigations would be the extension of the universal-type representation of the refined CS theories with $A_{n}$ and $D_{n}$ algebras to the remaining algebras: the simply laced $E_{n}$ and the non-simply laced classical $\left(B_{n}, C_{n}\right)$ and exceptional $\left(F_{4}, G_{2}\right)$ algebras, with the final aim of setting up a connection of the corresponding refined CS theories with some (refined) topological strings.

The present paper embodies the first step in this direction: we present, for the first time, universal-type representations of the partition function of the refined CS theory with each of the remaining gauge groups. The transformation of these partition functions into those of some specific refined topological strings is under investigation [15]

In section 2 we present the new representation of the partition function of the refined CS theory for all simple Lie algebras. It is based on a new Lie-algebraic identity for the determinant of the symmetrized Cartan matrix (the refined version of that in the [16]), and generalizes a feature of the non-refined theory, exploited in [3] earlier, which states that the partition function is equal to 1 when the coupling of CS is 0 .

Then, in section 6 we present this partition function in a "universal" form, which means that instead of the roots and other standard characteristics of a gauge algebra it now writes in terms of the Vogel's parameters. Simultaneously, the range of the refinement parameter is extended to include non-integer values, too.

We discuss the prospects of the further transformation of this partition function into partition functions of refined topological strings in Conclusion.

\footnotetext{
${ }^{1}$ We shall omit mentioning $S^{3}$ from now on, since we don't consider theories on other manifolds in this paper.
} 


\section{The partition function of the refined CS theory on $S^{3}$}

The partition function of CS theory on $S^{3}$ sphere was given in Witten's seminal paper [1] as the $S_{00}$ element of the $S$ matrix of modular transformations. For an arbitrary gauge group it is (see, e.g. $[2,17]$ )

$$
Z(k)=\operatorname{Vol}\left(Q^{\vee}\right)^{-1}\left(k+h^{\vee}\right)^{-\frac{r}{2}} \prod_{\alpha_{+}} 2 \sin \pi \frac{(\alpha, \rho)}{k+h^{\vee}}
$$

Here the so called minimal normalization of the invariant scalar product $($,$) in the root$ space is used, which implies that the square of the long roots equals 2. Other notations are: $\operatorname{Vol}\left(Q^{\vee}\right)$ is the volume of the fundamental domain of the coroot lattice $Q^{\vee}$, the integer $k$ is the CS coupling constant, $h^{\vee}$ is the dual Coxeter number of the algebra, $r$ is the rank of the algebra, the product is taken over all positive roots $\alpha_{+}$.

$\operatorname{Vol}\left(Q^{\vee}\right)$ is equal to the square root of the determinant of the matrix of scalar products of the simple coroots, accordingly for the simply laced algebras, in the minimal normalization, it is equal to the square root of the determinant of the Cartan matrix:

$$
\begin{aligned}
\operatorname{Vol}\left(Q^{\vee}\right) & =\left(\operatorname{det}\left(\alpha_{i}^{\vee}, \alpha_{j}^{\vee}\right)\right)^{1 / 2} \\
\alpha_{i}^{\vee} & =\alpha_{i} \frac{2}{\left(\alpha_{i}, \alpha_{i}\right)}, i=1, \ldots, r
\end{aligned}
$$

The same formula for the partition function, rewritten in an arbitrary normalization of the scalar product [2], is

$$
Z(\kappa)=\operatorname{Vol}\left(Q^{\vee}\right)^{-1}(\delta)^{-\frac{r}{2}} \prod_{\alpha_{+}} 2 \sin \pi \frac{(\alpha, \rho)}{\delta}
$$

where $k$ is now replaced by $\kappa, h^{\vee}$ by $t$, and $\delta=\kappa+t$. In this form the r.h.s. is invariant w.r.t. the simultaneous rescaling of the scalar product, $\kappa$, and $t$ (and hence $\delta$ ). In the minimal normalization they accept their usual values in (2.1).

In [3] it was noticed, that from this formula for the partition function one can derive an interesting closed expression for $\operatorname{Vol}\left(Q^{\vee}\right)$, which agrees with that in the Kac-Peterson's paper [16], (see eq. (4.32.2)), provided

$$
Z(0)=1
$$

This equality is completely natural from the physical point of view. Indeed, the CS theory is based on the unitary integrable representations of affine Kac-Moody algebras. At a given $k$ there is a finite number of such representations, and at $k=0$ there is not any non-trivial one.

So, from (2.4) and (2.5) we have

$$
\operatorname{Vol}\left(Q^{\vee}\right)=t^{-\frac{r}{2}} \prod_{\alpha_{+}} 2 \sin \pi \frac{(\alpha, \rho)}{t}
$$

which, as mentioned, agrees with [16]. Below we generalize this equation by inclusion of a refinement parameter. 
The generalization of the usual CS to the refined CS theory is given in [18-20]. It is based on the Macdonald's deformation of e.g. the Shur polynomials, and other "deformed" formulae, given in [21-23]. In a nutshell, the Macdonald's deformation yields the deformed $S$ and $T$ matrices of the modular transformations, and since these matrices define all observables in CS theory, one can naturally consider the "deformed" or the refined versions of all observables, i.e. the link/manifold invariants.

Particularly, the partition function of the refined CS theory on $S^{3}$ is given [18] by the $S_{00}$ element of the refined $S$-matrix. In [18] an orthogonal, instead of an orthonormal basis is used sometimes. We shall use the orthonormal one only (as in [11]), so there is no difference between e.g. $S_{00}$ and $S_{0}^{0}$.

We suggest the following expression for $S_{00}$ for the refined CS theory:

$$
Z(\kappa, y)=\operatorname{Vol}\left(Q^{\vee}\right)^{-1} \delta^{-\frac{r}{2}} \prod_{m=0}^{y-1} \prod_{\alpha_{+}} 2 \sin \pi \frac{y(\alpha, \rho)-m(\alpha, \alpha) / 2}{\delta}
$$

We assume that now $\delta=\kappa+y t, y$ is the refinement parameter, which we consider to be a positive integer at this stage.

Although we could not find the $Z(\kappa, y)$ in this exactly form in the literature, however, the expression (2.7) complies with the known formulae in different limits, e.g. at $y=1$ it yields the corresponding formula for the non-refined case (2.4). It also coincides with the corresponding formulae for the refined CS theory in $[11,18,20]$ for $A_{n}, D_{n}$ algebras. The coefficient $(\alpha, \alpha) / 2$ in front of the summation parameter $m$ coincides with that in the constant term formulae in $[24,25]$. Actually for non-simply laced algebras one can introduce two refinement parameters, one for each length of the roots, see e.g. [24, 25]. However, we did not try to introduce a second parameter (and also are not aware of the physical interpretation of it), so below we consider them to be coinciding, so that we always have one refinement parameter.

The latter expression of the partition function is supported by the key feature of (2.7): at $\kappa=0$ the equality $Z(0, y)=1$ holds, which is ensured by the following generalization of the formula (2.6) for the same object $\operatorname{Vol}\left(Q^{\vee}\right)$ :

$$
\operatorname{Vol}\left(Q^{\vee}\right)=(t y)^{-\frac{r}{2}} \prod_{m=0}^{y-1} \prod_{\alpha_{+}} 2 \sin \pi \frac{y(\alpha, \rho)-m(\alpha, \alpha) / 2}{t y}
$$

For $A_{n}$ algebras this equality can be easily proved with the use of the following wellknown identity, valid at an arbitrary positive integer $N$ :

$$
N=\prod_{k=1}^{N-1} 2 \sin \pi \frac{k}{N}
$$

Similarly it can be checked for all the remaining root systems, too.

Next, with (2.8) taken into account, we obtain the following expression of the partition function:

$$
Z(\kappa, y)=\left(\frac{t y}{\delta}\right)^{\frac{r}{2}} \prod_{m=0}^{y-1} \prod_{\alpha_{+}} \frac{\sin \pi \frac{y(\alpha, \rho)-m(\alpha, \alpha) / 2}{\delta}}{\sin \pi \frac{y(\alpha, \rho)-m(\alpha, \alpha) / 2}{t y}}
$$

which explicitly satisfies $Z(0, y)=1$, since $\delta=t y$ at $\kappa=0$. 


\section{Integral representation of the partition function for the refined CS theories}

For this purpose we apply the transformation, introduced in [3]. We transform each of the sines into a pair of Gamma-functions by the following well-known identity

$$
\frac{\sin \pi z}{\pi z}=\frac{1}{\Gamma(1+z) \Gamma(1-z)}
$$

and make use of the integral representation of (the logarithm of) the $\Gamma$ function:

$$
\ln \Gamma(1+z)=\int_{0}^{\infty} d x \frac{e^{-z x}+z\left(1-e^{-x}\right)-1}{x\left(e^{x}-1\right)}
$$

Let us rewrite the partition function in the following form:

$$
\begin{aligned}
Z(\kappa, y)= & \left(\frac{t y}{\delta}\right)^{y \frac{d i m-r}{2}+\frac{r}{2}} \prod_{m=0}^{y-1} \prod_{\alpha_{+}} \frac{\sin \pi \frac{y(\alpha, \rho)-m(\alpha, \alpha) / 2}{\delta}}{\pi \frac{y(\alpha, \rho)-m(\alpha, \alpha) / 2}{\delta}} \\
& \times \prod_{m=0}^{y-1} \prod_{\alpha_{+}} \frac{\pi \frac{y(\alpha, \rho)-m(\alpha, \alpha) / 2}{\sin } \pi \frac{y(\alpha, \rho)-m(\alpha, \alpha) / 2}{t y}}{\equiv} \\
\equiv & \left.\frac{t y}{\delta}\right)^{y \frac{d i m-r}{2}+\frac{r}{2}} Z_{1} Z_{2}
\end{aligned}
$$

and apply the abovementioned transformation to the first couple of products of sines (then similarly to the second couple of products):

$$
\begin{aligned}
\ln Z_{1} & =\ln \left(\prod_{m=0}^{y-1} \prod_{\alpha_{+}} \frac{\sin \pi \frac{y(\alpha, \rho)-m(\alpha, \alpha) / 2}{\delta}}{\pi \frac{y(\alpha, \rho)-m(\alpha, \alpha) / 2}{\delta}}\right) \\
& =-\int_{0}^{\infty} \frac{d x}{x\left(e^{x}-1\right)} \sum_{m=0}^{y-1} \sum_{\alpha_{+}}\left(e^{x \frac{y(\alpha, \rho)-m(\alpha, \alpha) / 2}{\delta}}+e^{-x \frac{y(\alpha, \rho)-m(\alpha, \alpha) / 2}{\delta}}-2\right)
\end{aligned}
$$

Let us introduce the following function for any simple Lie algebra $X$ of the rank $r$ :

$$
F_{X}(x, y)=r+\sum_{m=0}^{y-1} \sum_{\alpha_{+}}\left(e^{x(y(\alpha, \rho)-m(\alpha, \alpha) / 2)}+e^{-x(y(\alpha, \rho)-m(\alpha, \alpha) / 2)}\right)
$$

Then

$$
\sum_{m=0}^{y-1} \sum_{\alpha_{+}}\left(e^{x(y(\alpha, \rho)-m(\alpha, \alpha) / 2)}+e^{-x(y(\alpha, \rho)-m(\alpha, \alpha) / 2)}-2\right)=F_{X}(x, y)-r-y(\operatorname{dim}-r)
$$

and $\ln Z_{1}$ becomes

$$
\ln Z_{1}=-\int_{0}^{\infty} \frac{d x}{x\left(e^{x}-1\right)}\left(F_{X}\left(\frac{x}{\delta}, y\right)-r-y(\operatorname{dim}-r)\right)
$$

A similar transformation applies to $\ln Z_{2}$ :

$$
\ln Z_{2}=\int_{0}^{\infty} \frac{d x}{x\left(e^{x}-1\right)}\left(F_{X}\left(\frac{x}{t y}, y\right)-r-y(d i m-r)\right)
$$


and $\ln Z$ takes the form

$$
\ln Z=\frac{1}{2}(y(\operatorname{dim}-r)+r) \ln \left(\frac{t y}{\delta}\right)+\int_{0}^{\infty} \frac{d x}{x\left(e^{x}-1\right)}\left(F_{X}\left(\frac{x}{t y}, y\right)-F_{X}\left(\frac{x}{\delta}, y\right)\right)
$$

Finally, one can further transform this formula into an expression, similar to that which was derived in [12] for the non-refined theories.

Let us make the $x \rightarrow t y x / \delta$ rescaling in $\ln Z_{2}$, so that

$$
\ln Z_{2}=\int_{0}^{\infty} \frac{d x}{x\left(e^{t y x / \delta}-1\right)}\left(F_{X}\left(\frac{x}{\delta}, y\right)-r-y(\operatorname{dim}-r)\right) .
$$

Using the relation

$$
\frac{1}{e^{b x}-1}-\frac{1}{e^{a x}-1}=\frac{e^{a x}-e^{b x}}{\left(e^{a x}-1\right)\left(e^{b x}-1\right)}=\frac{\sinh \left(\frac{x(a-b)}{2}\right)}{2 \sinh \left(\frac{x a}{2}\right) \sinh \left(\frac{x b}{2}\right)},
$$

and making use of that the combined integrand is even under $x \rightarrow-x$, we can write $\ln Z$ as

$$
\begin{aligned}
\ln Z= & \frac{r+y(\operatorname{dim}-r)}{2} \log (t y / \delta) \\
& -\frac{1}{4} \int_{R_{+}} \frac{d x}{x} \frac{\sinh (x(t y-\delta))}{\sinh (x t y) \sinh (x \delta)}\left(F_{X}(2 x, y)-r-y(d i m-r)\right),
\end{aligned}
$$

where the integration range passes the origin by an infinitesimal semi-circle in the upper (or lower) half of the complex plane. We denote the corresponding contour $R_{+}$. We also took $x \rightarrow 2 x \delta$.

Due to the following identity

$$
\frac{1}{4} \int_{R_{+}} \frac{d x}{x} \frac{\sinh (x(t-\delta))}{\sinh (x t) \sinh (x \delta)}=-\frac{1}{2} \log \left(\frac{t}{\delta}\right)
$$

proved in [11] the integral of the $r+y(\operatorname{dim}-r)$ term in fact cancels against the log term in (3.15), so that we obtain the final expression:

$$
\ln Z=-\frac{1}{4} \int_{R_{+}} \frac{d x}{x} \frac{\sinh (x(t y-\delta))}{\sinh (x t y) \sinh (x \delta)} F_{X}(2 x, y)
$$

With a corresponding representation of $F_{X}(x, y)$ functions as a ratio of polynomials over $q=\exp x$, which is shown below in section 6 , the latter expression can be transformed into a product of multiple sine functions (see, e.g. [7, 12]), which then hopefully will make the further correspondence of it with the refined topological strings possible.

\section{The partition function of the refined CS for simply laced algebras}

In the non-refined case, i.e. at $y=1$ (when the sum over $m$ disappears), the partition function rewrites in terms of the Vogel's universal parameters. The corresponding $F_{X}(x, 1)$ 
coincides with the quantum dimension of the adjoint representation, which is the character $\chi_{a d}(x \rho)$, restricted to the $x \rho$ line, collinear with the Weyl vector $\rho$ :

$$
F_{X}(x, 1)=r+\sum_{\alpha_{+}}\left(e^{x(\alpha, \rho)}+e^{-x(\alpha, \rho)}\right)=\chi_{a d}(x \rho)
$$

So $F_{X}(x, y)$ can be called the refined quantum dimension.

The quantum dimension of the adjoint representation has been presented in the universal form in $[2,26]$ :

$$
\chi_{a d}(x \rho) \equiv f(x)=\frac{\sinh \left(x \frac{\alpha-2 t}{4}\right)}{\sinh \left(x \frac{\alpha}{4}\right)} \frac{\sinh \left(x \frac{\beta-2 t}{4}\right)}{\sinh \left(x \frac{\beta}{4}\right)} \frac{\sinh \left(x \frac{\gamma-2 t}{4}\right)}{\sinh \left(x \frac{\gamma}{4}\right)}
$$

Note that the notation $\alpha$ is used either for the root(s) of an algebra and for one of the Vogel's parameters. Since these objects are very different, hopefully no interpretation problem will appear.

Finally, the partition function in the non-refined case takes the following universal form

$$
Z(\kappa)=Z(\kappa, 1)=\left(\frac{t}{\delta}\right)^{\frac{d i m}{2}} \exp \left(-\int_{0}^{\infty} \frac{d x}{x\left(e^{x}-1\right)}\left(f\left(\frac{x}{\delta}\right)-f\left(\frac{x}{t}\right)\right)\right)
$$

first given in [3]

In the refined case there is not a similar universal answer for the double sum over $m$ and $\alpha_{+}$, however, for $A_{n}$ and $D_{n}$ algebras Krefl and Schwarz [11] have made a statement, equivalent to

$$
\sum_{m=0}^{y-1} \sum_{\alpha_{+}}\left(e^{x(y(\alpha, \rho)-m(\alpha, \alpha) / 2)}+e^{-x(y(\alpha, \rho)-m(\alpha, \alpha) / 2)}-2\right)=f(x, y)-\operatorname{dim}(y)
$$

with

$$
\begin{aligned}
f(x, y) & =\frac{\sinh \left(x \frac{\alpha-2 t y}{4}\right)}{\sinh \left(x \frac{\alpha}{4}\right)} \frac{\sinh \left(x y \frac{\beta-2 t}{4}\right)}{\sinh \left(x y \frac{\beta}{4}\right)} \frac{\sinh \left(x y \frac{\gamma-2 t}{4}\right)}{\sinh \left(x y \frac{\gamma}{4}\right)}, \\
\operatorname{dim}(y) & =\lim _{x \rightarrow 0} f(x, y)=y \operatorname{dim}-(y-1) \frac{(\beta-2 t)(\gamma-2 t)}{\beta \gamma} \\
f(x, 1) & =f(x)
\end{aligned}
$$

where it is assumed that $\alpha$ is the only negative parameter (equal to -2 in the minimal normalization of the scalar product).

The $\operatorname{dim}(y)$ can be further transformed. Indeed, consider the dimension formula for the simple Lie algebras:

$$
\operatorname{dim}=\frac{(\alpha-2 t)(\beta-2 t)(\gamma-2 t)}{\alpha \beta \gamma}=\frac{\alpha-2 t}{\alpha} \frac{(\beta-2 t)(\gamma-2 t)}{\beta \gamma}
$$

In the last expression both fractions are independent of the normalization. In the minimal normalization the first fraction is equal to $1+h^{\vee}$ (where $h^{\vee}$ is the dual Coxeter number) so we conclude that the second one is the rank of the algebra

$$
\frac{(\beta-2 t)(\gamma-2 t)}{\beta \gamma}=r
$$


since the following relation holds for all simply laced algebras:

$$
\operatorname{dim}=\left(1+h^{\vee}\right) r
$$

Finally, we have

$$
\operatorname{dim}(y)=y(\operatorname{dim}-r)+r
$$

With this relation we see that (4.4) is equivalent to

$$
F_{X}(x, y)=f(x, y)
$$

Then, with the use of (4.4), the partition function (3.12) becomes:

$$
Z(\kappa, y)=\left(\frac{t y}{\delta}\right)^{y \frac{d i m-r}{2}+\frac{r}{2}} \exp \left(-\int_{0}^{\infty} \frac{d x}{x\left(e^{x}-1\right)}\left(f\left(\frac{x}{\delta}, y\right)-f\left(\frac{x}{t y}, y\right)\right)\right)
$$

As mentioned, this result has been first proven for $A_{n}$ and $D_{n}$ series in [11]. In the next section we prove the relation (4.4) (and hence (4.12)) for the remaining simply laced algebras, namely, for $E_{n}$, thus generalizing (4.13) to the all simply laced simple Lie algebras.

\section{On validity of (4.4) for all simply laced algebras}

In this section we prove the statement of the previous section, i.e. generalize the relation (4.4) to all simply laced algebras.

The relation 4.4 tells that at an integer $y$ the polynomial in $q=e^{x}$ in the denominator of $f(x, y)$ divides that in the numerator. In [27] it is shown that the necessary condition for it to take place is that the power of each factor in the denominator should divide the power of one of the factors in the numerator. Indeed, this necessary condition is satisfied here. However, for a complete proof of 4.4 one should consider each algebra separately.

We claim that

$$
F_{X}(x, y)=f(x, y)
$$

for any simply laced Lie algebra $X$.

Take e.g. the $E_{6}$ algebra, for which the corresponding universal parameters in the minimal normalization are: $\alpha=-2, \beta=6, \gamma=8, t=12$. We should calculate the sum

$$
F_{E_{6}}(x, y)=6+\sum_{m=0}^{y-1} \sum_{\alpha_{+}} e^{x(y(\alpha, \rho)-m)}+e^{-x(y(\alpha, \rho)-m)}
$$

First note the number of roots $n_{L}$ with a given height $L=(\alpha, \rho)$ among all roots. The set of couples $\left(L, n_{L}\right)$ with a non-zero $n_{L}$ is

$$
\begin{aligned}
& (-11,1),(-10,1),(-9,1),(-8,2),(-7,3),(-6,3),(-5,4),(-4,5), \\
& (-3,5),(-2,5),(-1,6),(0,6),(1,6),(2,5),(3,5),(4,5),(5,4),(6,3), \\
& (7,3),(8,2),(9,1),(10,1),(11,1)
\end{aligned}
$$


which of course is symmetric w.r.t. the $L \leftrightarrow-L$. We also include the element $(0,6)$ in this list, which is just the first term 6 in (5.2). Then, using this data, we note that the sum in (5.2) is given by

$$
\begin{aligned}
F_{E_{6}} & =\phi(11 y)+\phi(8 y)+\phi(7 y)+\phi(5 y)+\phi(4 y)+\phi(y) \\
\phi(n) & =\sum_{i=-n}^{n} q^{i}=\frac{q^{2 n+1}-1}{q^{n}(q-1)} \\
q & =e^{x}
\end{aligned}
$$

Combining the sums $\phi(11 y)+\phi(8 y)+\phi(5 y)$ and $\phi(7 y)+\phi(4 y)+\phi(y)$, we get

$$
\begin{aligned}
\phi(11 y)+\phi(8 y)+\phi(5 y) & =\frac{\left(q^{9 y}-1\right)\left(q^{5 y+1}-q^{-11 y}\right)}{(q-1)\left(q^{3 y}-1\right)} \\
\phi(7 y)+\phi(4 y)+\phi(y) & =\frac{\left(q^{9 y}-1\right)\left(q^{y+1}-q^{-7 y}\right)}{(q-1)\left(q^{3 y}-1\right)} \\
F_{E_{6}} & =\frac{\left(q^{9 y}-1\right)}{(q-1)\left(q^{3 y}-1\right)}\left(q^{4 y}+1\right)\left(q^{y+1}-q^{-11 y}\right) \\
& =\frac{\left(q^{9 y}-1\right)\left(q^{8 y}-1\right)\left(q^{y+1}-q^{-11 y}\right)}{(q-1)\left(q^{3 y}-1\right)\left(q^{4 y}-1\right)}
\end{aligned}
$$

which can be easily checked to coincide with $f(x, y)$ for the universal parameters corresponding to $E_{6}$ algebra.

Literally similar calculations can be carried out for the remaining $E_{7}, E_{8}$ algebras, as well as for Krefl-Schwarz cases $A_{n}, D_{n}$, leading to the same conclusion.

\section{A universal-type presentation of the partition function for the non- simply laced algebras}

Equations (4.4), (5.2) do not hold for the non-simply laced algebras. However, one can present the corresponding sum in a similar form, appropriate for the further duality considerations $[3,8,12]$. The latter means that it can be presented as a ratio of a sum of exponents of $x$ (i.e. powers of $q=\exp x$ ) in the numerator and some sines in the denominator. So, we are aiming to represent $F_{X}$ as follows:

$$
F_{X}=r+\sum_{m=0}^{y-1} \sum_{\alpha_{+}}\left(e^{x(y(\alpha, \rho)-m(\alpha, \alpha) / 2)}+e^{-x(y(\alpha, \rho)-m(\alpha, \alpha) / 2)}\right)=\frac{A_{X}}{B_{X}}
$$

where $X$ denotes an algebra of type $B, C, F$ or $G, r$ is its rank, $B_{X}$ is a product of a number of terms of the form $q^{a}-1$, and $A_{X}$ is a polynomial in $q$.

One subtlety regarding the formulae (6.1), which makes them different from the (5.1), is that in (6.1) one should explicitly mention the normalization of the scalar product. In (5.1) both sides are invariant under the rescaling of the scalar product in the l.h.s. (with corresponding rescaling of the universal parameters in the r.h.s.), and the simultaneous appropriate rescaling of $x$. However, in (6.1) a similar rescaling of the scalar product and $x$ leaves invariant only the l.h.s., whilst the ratio $A_{X} / B_{X}$ in the r.h.s. is dependent only of $x$, 
thus changes under its rescaling. This means that when substituting the r.h.s. of (6.1) into the partition function 3.12 one should take the parameters $t$ and $\delta$ in the same normalization. The normalizations below are chosen to avoid the appearance of fractional powers of $q$.

Now we present $F_{X}$ for all non-simply laced algebras.

Let us consider the $B_{n}$ algebras. Normalization corresponds to $\alpha=-4$, i.e. the square of the long root is 4 . The corresponding representation we mentioned above is

$$
\begin{aligned}
F_{B_{n}}(x, y)= & \frac{A_{B_{n}}}{B_{B_{n}}} \\
A_{B_{n}}= & q^{4 n y+2}+q^{-4(n-1) y} \\
& +(q+1)\left(q^{y}-1\right)\left(q^{2 y}+1\right)\left(q^{2 n y}-1\right)\left(q^{y-2 n y}+q\right)-q^{4 y}-q^{2} \\
B_{B_{n}}= & \left(q^{2}-1\right)\left(q^{4 y}-1\right),
\end{aligned}
$$

For the $C_{n}$ algebras we also choose the same normalization with the square of the long root being 4 . Then $F_{X}$ writes as

$$
\begin{aligned}
F_{C_{n}}= & \frac{A_{C_{n}}}{B_{C_{n}}} \\
B_{C_{n}}= & \left(q^{2}-1\right)\left(q^{2 y}-1\right) \\
A_{C_{n}}= & (q+1) q^{y}\left(q^{2 n y}-1\right)\left(q^{2 n y+1}-1\right) \\
& +\left(q^{2 y}-1\right)\left(q^{n y}-1\right)\left(q^{n y+1}-1\right)\left(q^{2 n y+1}-1\right)
\end{aligned}
$$

For the $F_{4}$, with the same normalization, we have

$$
\begin{aligned}
F_{F_{4}}= & \frac{A_{F_{4}}}{B_{F_{4}}} \\
B_{F_{4}}= & \left(q^{2}-1\right) \\
A_{F_{4}}= & q^{-16 y}\left(q^{2 y}+1\right)\left(-q^{2 y}+q^{4 y}+1\right)\left(q^{12 y+1}-1\right) \\
& \times\left(q^{5 y+1}-q^{8 y+1}+q^{9 y+1}+q^{14 y+1}+q^{5 y}-q^{6 y}+q^{9 y}+1\right)
\end{aligned}
$$

For the $G_{2}$ we use the normalization corresponding to the square of the long root to be equal to 6 . The corresponding $F_{G_{2}}$ function is

$$
\begin{aligned}
F_{G_{2}}= & \frac{A_{G_{2}}}{B_{G_{2}}} \\
B_{G_{2}}= & q^{3}-1 \\
A_{G_{2}}= & q^{-9 y}\left(q^{6 y+1}-1\right) \\
& \times\left(q^{4 y+1}+q^{8 y+1}+q^{4 y+2}-q^{6 y+2}+q^{8 y+2}+q^{12 y+2}+q^{4 y}-q^{6 y}+q^{8 y}+1\right)
\end{aligned}
$$

\section{Conclusion}

By closing the contour of integration in (3.18) in the upper semi plane, one obtains the output by means of a sum of contributions of poles. As it was shown in a number of 
cases in $[3,7,12]$, the contribution of the so-called perturbative poles, i.e. those coming from $\sinh (x \delta)$, exactly coincides with the Gopakumar-Vafa partition function of the corresponding dual topological string. The corresponding contribution with the use of the newly derived expressions $F_{X}$ should be examined next, aiming at the interpretation of the initial partition function in terms of some (refined) topological strings, indeed if such strings exist. Obviously, that string would be the candidate for a dual description of the corresponding CS theory.

We also hope that the exact closed expressions for the partition functions of the refined CS theory with an arbitrary gauge group, derived in the present paper, will find other applications, too.

\section{Acknowledgments}

The work of MA was fulfilled within the Regional Doctoral Program on Theoretical and Experimental Particle Physics sponsored by VolkswagenStiftung. MA and RM are partially supported by the Science Committee of the Ministry of Science and Education of the Republic of Armenia under contracts 20AA-1C008, 21AG-1C060.

Open Access. This article is distributed under the terms of the Creative Commons Attribution License (CC-BY 4.0), which permits any use, distribution and reproduction in any medium, provided the original author(s) and source are credited.

\section{References}

[1] E. Witten, Quantum field theory and the Jones polynomial, Commun. Math. Phys. 121 (1989) 351 [INSPIRE].

[2] R.L. Mkrtchyan and A.P. Veselov, Universality in Chern-Simons theory, JHEP 08 (2012) 153 [arXiv: 1203.0766] [INSPIRE].

[3] R.L. Mkrtchyan, Nonperturbative universal Chern-Simons theory, JHEP 09 (2013) 054 [arXiv: 1302.1507] [INSPIRE].

[4] P. Vogel, Algebraic structures on modules of diagrams, preprint, http://www.math.jussieu.fr/ vogel/diagrams.pdf, (1995) [J. Pure Appl. Alg. 215 (2011) 1292].

[5] P. Vogel, The universal Lie algebra, preprint, https://webusers.imj-prg.fr/ pierre.vogel/grenoble-99b.pdf, (1999).

[6] J.M. Landsberg and L. Manivel, A universal dimension formula for complex simple Lie algebras, Adv. Math. 201 (2006) 379.

[7] R.L. Mkrtchyan, On a Gopakumar-Vafa form of partition function of Chern-Simons theory on classical and exceptional lines, JHEP 12 (2014) 171 [arXiv:1410.0376] [INSPIRE].

[8] R.L. Mkrtchyan, Chern-Simons theory with the exceptional gauge group as a refined topological string, Phys. Lett. B 808 (2020) 135692 [arXiv:2007.09346] [INSPIRE].

[9] P. Deligne, La série exceptionnelle des groupes de Lie (in French), C. R. Acad. Sci. Paris 322 (1996) 321. 
[10] A.M. Cohen and R. de Man, Computational evidence for Deligne's conjecture regarding exceptional Lie groups, C. R. Acad. Sci. Paris 322 (1996) 427.

[11] D. Krefl and A. Schwarz, Refined Chern-Simons versus Vogel universality, J. Geom. Phys. 74 (2013) 119 [arXiv: 1304.7873] [INSPIRE].

[12] D. Krefl and R.L. Mkrtchyan, Exact Chern-Simons/topological string duality, JHEP 10 (2015) 045 [arXiv : 1506.03907] [INSPIRE].

[13] Y. Hatsuda, M. Mariño, S. Moriyama and K. Okuyama, Non-perturbative effects and the refined topological string, JHEP 09 (2014) 168 [arXiv:1306.1734] [INSPIRE].

[14] Y. Hatsuda, Spectral zeta function and non-perturbative effects in ABJM Fermi-gas, JHEP 11 (2015) 086 [arXiv: 1503.07883] [INSPIRE].

[15] M.Y. Avetisyan and R.L. Mkrtchyan, in preparation.

[16] V.G. Kac and D.H. Peterson, Infinite dimensional Lie algebras, theta functions and modular forms, Adv. Math. 53 (1984) 125 [INSPIRE].

[17] P. Di Francesco, P. Mathieu and D. Sénéchal, Conformal field theory, Springer-Verlag, New York, NY, U.S.A. (1997).

[18] M. Aganagic and S. Shakirov, Knot homology and refined Chern-Simons index, Commun. Math. Phys. 333 (2015) 187 [arXiv:1105.5117] [INSPIRE].

[19] M. Aganagic and S. Shakirov, Refined Chern-Simons theory and knot homology, Proc. Symp. Pure Math. 85 (2012) 3 [arXiv: 1202.2489] [INSPIRE].

[20] M. Aganagic and K. Schaeffer, Orientifolds and the refined topological string, JHEP 09 (2012) 084 [arXiv: 1202.4456] [INSPIRE].

[21] I.G. Macdonald, A new class of symmetric functions, in Actes 20-e Seminaire Lotharingen, Publ. I.R.M.A., Strasbourg, France (1988), pg. 131.

[22] I.G. Macdonald, Orthogonal polynomials associated with root systems, preprint, (1988).

[23] I.G. MacDonald, Some conjectures for root systems, SIAM J. Math. Anal. 13 (1982) 988.

[24] I. Cherednik, Macdonald's evaluation conjectures and difference Fourier transform, q-alg/9412016.

[25] I. Cherednik, Double affine Hecke algebras and Macdonald's conjectures, Ann. Math. 141 (1995) 191.

[26] B. Westbury, Invariant tensors and diagrams, in Proceedings of the tenth Oporto meeting on geometry, topology and physics, (2001) [Int. J. Mod. Phys. A 18 (2003) 49].

[27] M.Y. Avetisyan and R.L. Mkrtchyan, Universality and quantum dimensions, Phys. Part. Nucl. Lett. 17 (2020) 784 [INSPIRE].

[28] G. Lockhart and C. Vafa, Superconformal partition functions and non-perturbative topological strings, JHEP 10 (2018) 051 [arXiv: 1210.5909] [INSPIRE]. 